\title{
Claves para la disminución de la huella de carbono en el turismo: Caso Cuba
}

Keys to decrease the carbon footprint in tourism: The Case of Cuba Lisandra Torres Hechavarría. ${ }^{1} \&$ Alejandro Delgado Castro. ${ }^{2}$

\begin{abstract}
This research aims at determining key actions for a low carbon tourism development in the current Cuban context. To fulfil the objective, tourists' carbon footprint was estimated, as well as the factors and actors of influence in diminishing it. The methodological process carried out included a comparative analysis of carbon footprint measurement to propose a new one meeting the data availability. Data were obtained from international referents, given the lack of tourism carbon emissions data in the studied destination. The formula produced allowed to estimate tourists' carbon footprint in Havana, which was taken as a reference. To determine influencing factor, it was analysed the Tourism Pentagonal Model and the Convergent Socioeconomic Actors Theory for destinations' sustainability. It was also taken in consideration the Cuban context and Cuba's environmental international commitments. It was also examined the applicability of international organisms' guides and recommendations. Current market structure, actors' ignorance of the phenomenon, and the economic, commercial and financial blockade of the United States of America, are the main factors influencing current tourism carbon footprint in Cuba. Main action that can be implemented in short term is capacity building for all tourism actors.
\end{abstract}

Key Words: Carbon Footprint, Tourism, Cuba.

\section{Resumen}

\footnotetext{
${ }^{1}$ Doctora en Ciencias Económicas. Facultad de Turismo, Universidad de La Habana. lisandra_torres@ftur.uh.cu

2 Doctor en Ciencias Económicas. Facultad de Turismo, Universidad de La Habana. alejandro_delgado@ftur.uh.cu
} 
El presente trabajo se propone determinar acciones clave para un desarrollo turístico bajo en emisiones de carbono de acuerdo al contexto actual cubano. Para cumplir el objetivo, se estimó la huella de carbono promedio actual de los turistas y se identificaron los factores que intervienen en su disminución. El proceso metodológico de la investigación empleó el análisis comparativo de las metodologías de medición de la huella de carbono para determinar una propia de acuerdo a los datos disponibles, los cuales se obtuvieron a partir de referentes internacionales al no estar disponibles en el destino. La fórmula ideada permitió estimar la huella de carbono actual del turismo en la Habana como referente. En la identificación de factores que intervienen en la disminución de la huella de carbono se analizó el Modelo Pentagonal del sector turístico y la teoría de actores socioeconómicos convergentes para la sostenibilidad de un destino. Se tuvieron en cuenta el contexto cubano, los compromisos internacionales sobre medio ambiente firmados por Cuba y se evaluó la aplicabilidad de buenas prácticas, guías y recomendaciones hechas por organismos internacionales. Se determinó que los principales elementos que influyen en la huella de carbono del turismo en Cuba son su estructura de mercados, el desconocimiento de los actores con respecto al fenómeno y el bloqueo económico, financiero y comercial de Estados Unidos. Además de lo anterior, la principal acción que puede acometerse en el corto plazo es la capacitación a los diferentes actores que intervienen en el turismo. En aras de lograr el propósito trazado se consultaron diversas fuentes bibliográficas, seleccionándose las herramientas "Análisis de las cinco fuerzas competitivas" y "Análisis de la cadena de Valor" para la valoración del Grupo Gaviota S.A. En el análisis de la posición del Grupo Gaviota en el sector hotelero enfocado a la modalidad de ciudad, se empleó el Método Delphi. Además se realizó un análisis de las ventajas competitivas que posee Gaviota S.A operando bajo la forma de administración: Contrato de Gestión. Se realizaron entrevistas a los mandos de primer nivel en los hoteles seleccionados para determinar los elementos que aportan valor a la cadena. Entre los principales resultados se encuentran los Factores Claves de Éxito, determinados por las Capacidades Distintivas y las Ventajas Competitivas, así como las posibles estrategias para aumentar su situación competitiva.

Palabras Clave: Huella De Carbono, Turismo, Cuba

\section{Introducción}

Desde 1990 la emisión de gases de efecto invernadero (GEI) de larga duración causantes del calentamiento global y el cambio climático, han aumentado en un 43\% hasta el 2018. El dióxido de carbono ( $\mathrm{CO} 2$ ) es responsable por el $80 \%$ de ese incremento como resultado de la quema de combustibles fósiles por lo que es considerado el GEI de mayor impacto en el cambio climático (ONU, 2019). Esta situación ha provocado la generación de varios instrumentos internacionales para tomar acción con respecto al cambio climático: la Convención Marco de las Naciones Unidas sobre Cambio Climático (CMNUCC) firmada en 1992 por 197 países, el Protocolo de Kyoto firmado en 1995 por 192 países que consideraba acciones en dos etapas 2002-2012 y 2013-2020; y el Acuerdo de París 
firmado por 195 naciones en 2016, para el cual se analizaron los avances realizados por cada país en la reducción de las emisiones de carbono de acuerdo a sus compromisos en la Cumbre sobre Cambio Climático 2019. Aunque el cambio climático es un proceso natural, la aceleración del calentamiento global ha sido en gran medida causada por las actividades humanas.

El turismo como actividad económica ha crecido de forma continua en las últimas décadas. El aumento en el número de personas que se desplazan desde sus países o regiones de origen a otra, ha causado impactos económicos, sociales y medioambientales en los destinos turísticos, los cuales han sido objeto de investigación. La relación entre el desarrollo del turismo y el medio ambiente natural tiene dos aristas principales. Por un lado, los recursos naturales y antrópicos son los activos fundamentales de un destino turístico; pero por otro lado, el desarrollo del turismo puede tener un impacto negativo sobre el medio ambiente, especialmente en la generación de GEI asociados a la movilidad internacional (Khoo-Lattimore y Prideaux, 2013; Lund-Durlacher y Dimanche, 2013; Moscardo et al., 2013). Los estudios de Filimonau et al. (2014) muestran que el transporte hasta el destino genera la huella de carbono más grande en el turismo, aun cuando, en dependencia del tiempo de duración de la estancia del turista, los productos que consumen como parte de sus vacaciones pueden hacer una contribución de carbono que logra incluso superar el porcentaje del elemento de tránsito. De forma general se considera que el turismo es el causante de al menos el 5\% de las emisiones de GEI (Wang, 2017). Lo anterior ha hecho indispensable pensar en una forma de disminuir la huella de carbono del turismo, por lo que se han producido varias iniciativas, investigaciones y guías para el desarrollo de un turismo bajo en emisiones de carbono (OMT y OEA, 2018; Coronado et al., 2014; Huang y Deng, 2011; Yang, 2010).

Cuba es un país que ha sido firmante de todos los instrumentos y convenciones internacionales con respecto a la disminución de las emisiones de carbono. En el Acuerdo de París, Cuba se comprometió a realizar un esfuerzo en la disminución de sus emisiones de GEI, sin embargo esto depende en gran medida de la posibilidad de cooperación internacional en el tema dadas las condiciones económicas y el bloqueo que sufre el país. Hasta el momento no se ha encontrado en la literatura científica evidencias de la medición de la huella de carbono del turismo en el país o una aproximación a su estimación, lo cual constituiría un paso importante para gestionar su disminución. El Gobierno cubano ha adoptado varias medidas para disminuir las emisiones de GEI, sin embargo hasta el momento desde el sector turístico no se ha proyectado un modelo de desarrollo turístico bajo en emisiones de carbono. Además de lo anterior, cualquier política o modelo para el sector turístico cubano debe estar en consonancia con las realidades del país. Por lo anterior se han determinado las siguientes preguntas de investigación: ¿Cuál es la huella de carbono actual por turista en Cuba? ¿Cuáles son los factores y actores que inciden en la disminución de la huella de carbono en el turismo en Cuba? Teniendo en cuenta los compromisos internacionales asumidos por Cuba con respecto a este tema, ¿qué acciones pudieran acometerse en el sector turístico de acuerdo a la situación actual del país? 
De acuerdo a lo anterior la presente investigación se plantea como objetivo identificar acciones clave para un desarrollo turístico bajo en emisiones de carbono de acuerdo al contexto actual cubano. Las principales limitaciones de la investigación están dadas por ser una primera aproximación al tema en el contexto cubano, con la consiguiente restricción de datos de fuentes primarias con respecto a las emisiones de carbono del sector en Cuba por lo que la huella de carbono ha sido estimada solo para el destino La Habana, por demás los datos disponibles solo permitieron estimar la huella de turistas que viajan a La Habana por avión. No obstante lo anterior, se considera un punto de partida válido ya que el resto de los factores analizados se considera pueden validarse en el futuro para otros destinos cubanos.

\section{Turismo bajo en emisiones de carbono}

El turismo es un sector muy dependiente del medio ambiente y del clima de forma particular. El cambio climático y cómo los destinos se enfrentan a él, se está convirtiendo cada día en un factor más relevante para las decisiones de viajes y la competitividad turística de los destinos (Yang, 2010). El turismo bajo en emisiones de carbono es una propuesta de principios del siglo XXI, que propone la relación producción-consumo en base a los elementos de la economía baja en emisiones de carbono. La teoría relacionada con este último concepto fue desarrollada en el documento "Nuestra Energía Futura-Para crear una Economía baja en Emisiones de carbono" publicado por los británicos en 2003 (Can y Hongbing, 2011). De acuerdo a Chen (2010) la economía baja en emisiones de carbono se define como un modelo económico, método de consumo energético y estilo de vida con elevada eficiencia, productividad y bajos consumos, emisiones y contaminación. Su esencia es la eficiencia energética, el desarrollo de energías limpias y la búsqueda de un Producto Interno Bruto "Verde" a través de la innovación tecnológica, el cambio estructural de la industria, y la transformación de las ideas de las personas con respecto a su supervivencia y desarrollo con el apoyo de un marco regulatorio consecuente. Yuan et al. (2011) manifiestan que una economía baja en emisiones de carbono es la primera fase de un desarrollo económico bajo en emisiones de $\mathrm{CO}$, para la cual es indispensable un cambio en los modos de actuación de la industria y el turismo teniendo en cuenta sus relaciones biunívocas.

Luo y Zhang (2011) y Zhang et al. (2016) coinciden en que un turismo bajo en emisiones de carbono se enfoca en el comportamiento de los seres humanos, entendido como cambios en el diseño y gestión de los productos turísticos así como en los patrones o comportamiento de consumo de los turistas, por lo que incluye todas las etapas el proceso de producción-consumo. En el camino a esos objetivos, algunas herramientas son fundamentales para supervisar y gestionar la disminución en las emisiones entre las que se encuentran la medición de la huella de carbono, el etiquetado de carbono y las certificaciones. 
El desarrollo de un turismo bajo en emisiones de carbono precisa desde el punto de la demanda un cambio en los patrones y hábitos de consumo de los turistas hacia actividades y destinos turísticos con una baja huella de carbono. Desde la oferta deben producirse cambios en cuatro elementos fundamentales:

- Creación de infraestructura turística baja en emisiones de carbono (transporte, alojamientos, restaurantes, tiendas de suvenires, entre otros) (Tang et al, 2011),

- $\quad$ Empleo de productos y trabajadores locales (alimentos, bebidas, suvenires, personal de servicio, entre otros) (Tang et al, 2011; Qi y Hong, 2011),

- Diseño de productos o atracciones turísticas bajas en emisiones de carbono (Tang et al, 2011; Changbo y Jingjing, 2011),

- $\quad$ Protección del medio ambiente del destino turístico a través de la creación de sumideros de carbono (Tang et al, 2011; Can y Hongbing, 2011).

Hasta el momento se han realizado varias experiencias que sirven de guía para la puesta en práctica del turismo bajo en emisiones de carbono en entornos rurales y urbanos (Ningrum et al, 2017; Pina et al, 2017; 2018). En Vietnam, en las ciudades de Chiang Mai y Hue se han propuesto acciones para el desarrollo del turismo urbano bajo en emisiones de carbono. En el caso de Chiang Mai, consisten en un cambio tecnológico del transporte interno individual en la ciudad hacia vehículos no motorizados o transporte público, ya que la aviación se salía del campo de acción de la ciudad (Kumar et al., 2016). Desde el punto de vista socieconómico, se apreció que la restricción de movimientos en la ciudad, tuvo un impacto en el aumento del consumo de productos locales. La ciudad de Hue tiene un patrimonio cultural vasto, percibido en sus edificios e infraestructuras antiguos, por lo que era necesario integrar la visión histórico-cultural a las medidas que se tomaran. En este caso se aumentó o rescataron las casas-jardines, una edificación típica de la ciudad, en las que se crean pequeños ecosistemas que actúan como sumideros de carbono, generadores de compost o alimento animal, lo que reduce los efectos de la transportación al no tener que importarlos. Además de lo anterior tuvo un impacto social y económico pues se establecieron nuevos empleos y aumentaron los ingresos por turismo en la comunidad por la creación de nuevos atractivos (Kumar et al., 2016). En Latinoamérica existen evidencias de trabajos en Bolivia, Nicaragua, Colombia y Perú desde fecha anterior a 2014 con respecto a medidas de adaptación y mitigación al cambio climático entre las que se encontraban la disminución de la huella de carbono, pero sin abordar el tema del turismo. Específicamente en turismo, en el año 2018 se produjo el documento "El turismo y los Objetivos de Desarrollo Sostenible: Buenas prácticas en las Américas" (OMT y OEA, 2018) en el cual se recogen experiencias turísticas exitosas sin embargo no se menciona ninguna específica de turismo bajo en carbono por lo que puede entenderse como un tema que aún no ha sido tratado en profundidad en la región.

\section{La huella de carbono y su evaluación}


Se estima que el turismo tiene uno de los más grandes impactos directos e indirectos en el cambio climático, contribuyendo al 5\% de las emisiones globales de CO2 en 2005, con aproximadamente un $8 \%$ de contribución al forzamiento radiativo (incluido el impacto de los gases de efecto invernadero de corta y larga vida) sobre el calentamiento global (OMT, 2008). En este contexto, el análisis de la huella de carbono se centra en cuestiones medioambientales causadas por el turismo, particularmente el consumo de energía y emisiones de $\mathrm{CO} 2$.

La huella de carbono es el total de emisiones de $\mathrm{CO} 2$ causadas por una organización, producto o persona; las etiquetas y certificaciones de carbono, están estrechamente relacionadas con las mediciones de la huella de carbono dado que las etiquetas muestran las emisiones de $\mathrm{CO} 2$ de un producto o servicio durante todo su ciclo de vida y las certificaciones inciden en la imagen del producto o servicio, comunicando su responsabilidad ambiental e base a su huella de carbono y otros factores relacionados. Las emisiones de dióxido de carbono, se definen como la cantidad de dióxido de carbono equivalente (CO2-eq) liberado a la atmósfera directa e indirectamente por la actividad turística. Estas han sido ampliamente investigadas a varias escalas, como los análisis para los países de Nueva Zelanda (Becken y Patterson, 2006), Suecia (Gössling y Hall, 2008), Poole (Filimonau et al 2011), la región Gales (Munday et al., 2013), España (Cadarso et al., 2015; 2016) y la isla de Taiwan (Tsai y Lin, 2018; Yu y Tsung-Lin, 2019). En el estudio de la huella de carbono del turismo sobresale la evaluación del impacto de las emisiones de gases de efecto invernadero del sector en el cambio climático (Wang et al, 2017).

No existe en estos momentos una metodología generalizada en la comunidad científica para la evaluación de la huella de carbono de la actividad turística (Wang et al, 2017). Se ha determinado que puede evaluarse a partir de los impactos directos e indirectos de las visitas de turistas. Entre los impactos directos se encuentran las emisiones del transporte aéreo, marítimo o terrestre hasta el destino y en este, el alojamiento, las visitas a atracciones, el consumo de alimentos y bebidas, los productos para la venta minorista a los turistas, las emisiones derivadas de la comercialización de los productos turísticos así como otros servicios en el destino.

\section{Metodología}

La investigación se estructuró de la siguiente forma: 1.- Medición (estimada) de la huella de carbono por turista en La Habana, 2.- Identificación de factores y actores que intervienen en la disminución de la huella de carbono, 3.- Contextualización de las buenas prácticas internacionales a la realidad cubana. Se ha escogido La Habana por ser un destino complejo, urbano, visitado por más del $70 \%$ de los turistas que viajan al país y donde se encuentra el aeropuerto internacional más importante de la isla.

\section{Medición (estimada) de la huella de carbono por turista en La Habana}


El análisis comparativo de varias metodologías de evaluación permitió determinar que para los efectos de este estudio, se ha estimado solamente la huella de carbono directa y se ha calculado teniendo en cuenta los vuelos directos desde los países emisores, el alojamiento de acuerdo a la estancia media en el destino, así como la huella promedio para otras actividades en destinos de ciudad utilizando las siguientes fórmulas:

$$
\begin{aligned}
& \mathrm{tCO}_{2} \mathrm{eq}_{\mathrm{Mi}}=\left[\mathrm{tCO}_{2 \text { Vuelo }}+\left(\mathrm{tCO}_{2 \text { Aloj }}+\mathrm{tCO}_{2 \text { Otros }}\right) * \mathrm{E}\right]^{*} \text { pax } \\
& \mathrm{tCO}_{2} \mathrm{eq}_{\mathrm{D}}=\sum \mathrm{Mi}
\end{aligned}
$$

En las cuales:

$\mathrm{tCO}_{2}$ eq: Toneladas equivalentes de Dióxido de Carbono (Huella de Carbono)

$\mathrm{tCO}_{2}$ Vuelo: Toneladas equivalentes de Dióxido de Carbono del vuelo a La Habana.

$\mathrm{tCO}_{2}$ Aloj: Toneladas equivalentes de Dióxido de Carbono en Alojamiento

$\mathrm{tCO}_{2}$ Otros: Toneladas equivalentes de Dióxido de Carbono en otras actividades en el destino

E: Estancia media en el destino.

Mi: Mercados emisores a La Habana.

Pax: Número de pasajeros a La Habana en un año.

La fórmula 1 muestra el cálculo de la huella de un mercado emisor a un destino específico, mientras que la fórmula 2 permite el cálculo de la huella de carbono total de los turistas en un destino turístico.

En el caso específico de los cubanos residentes en el exterior, las estadísticas turísticas no recogen con claridad los países de procedencia. Además de lo anterior el comportamiento de estos en el destino no coincide con el del resto de los mercados, pues su alojamiento y hábitos de compra en general difieren en buena medida de los demás turistas por lo que no se han incorporado en los cálculos. La estimación de la huella de carbono de los cubanos residentes en el exterior en sus visitas a Cuba pudiera ser motivo de una investigación específica al igual que un cálculo más exacto de la huella de carbono de los turistas, teniendo en cuenta las mediciones reales de la huella en el destino La Habana y sus efectos indirectos. Se emplearon como referentes para la medición datos de consumo de turistas en ciudades con características similares a La Habana dada la falta de datos reales (Rico et al., 2019)

\section{Identificación de factores y actores que intervienen en la disminución de la huella de carbono.}


En este paso se analizó el Modelo Pentagonal del sector turístico desarrollado por Martín (2010) en el que resumen los factores endógenos y exógenos que intervienen en el turismo desde un punto de vista estructural/funcional. En este se incluyen los factores de conversión de recursos en oferta turística que generan un flujo turístico, así como los elementos del macroentorno (Tecnología, Políticas generales y sectoriales, Leyes, Economía, Capital Humano) que inciden en ellos. Además de lo anterior, se analizó la Teoría de actores socioeconómicos convergentes para la sostenibilidad de un destino turístico propuesta en Torres (2017). Esta teoría propone que para lograr la sostenibilidad de un destino turístico, todos los actores convergentes en el deben beneficiarse, participar en la toma de decisiones y en las acciones para su desarrollo de forma integrada. Los actores principales en los destinos turísticos, de acuerdo con la autora antes mencionada, son los propios turistas, empresas privadas y públicas (no solo turísticas pues otras de apoyo garantizan la infraestructura y deben beneficiarse y participar también), la comunidad de acogida, el gobierno local, los medios de comunicación y otras instituciones afines como por ejemplo las universidades o centros de investigación.

Ambas teorías contribuyeron a identificar elementos necesarios a tener en cuenta para la propuesta de acciones de acuerdo al papel e influencia de los factores y actores en el destino. Para la obtención de información al respecto se realizó una revisión bibliográfica profunda y se entrevistaron especialistas del Ministerio de Turismo de la República de Cuba, los cuales aportaron información relevante al respecto. Las entrevistas a los especialistas fueron semiestructuradas con preguntas abiertas que permitieran obtener un mayor volumen de información, teniendo en cuenta que es un estudio exploratorio. Se aplicó un muestreo intencional dado que los especialistas consultados son los que tienen un conocimiento más profundo del sector por sus años de trabajo. Las entrevistas permitieron completar la información necesaria para conocer el estado del país con respecto a los elementos endógenos y exógenos del Modelo Pentagonal y la implicación actual de los actores de convergencia del destino en la disminución de la huella de carbono del turismo en el país.

\section{Contextualización de las buenas prácticas internacionales a la realidad cubana}

De acuerdo a los factores y actores derivados del paso anterior, se identificaron buenas prácticas en la gestión de la oferta y la demanda para la disminución de la huella de carbono del turismo a partir de publicaciones científicas de casos de estudio y guías presentadas por organismos internacionales. Se analizó por los autores de la presente investigación la posibilidad real de implementación de las buenas prácticas identificadas de acuerdo a la realidad cubana para la realización de la propuesta final. 


\section{Resultados del modelo análisis de la matriz del perfil competitivo.}

\section{Huella de carbono por turista en La Habana}

Los resultados de la medición de la huella de carbono estimada de los turistas en La Habana en 2018 por país emisor se muestran en la figura 1. La huella de carbono ha sido estimada en 1,09 toneladas de CO2eq por turista que visita La Habana y una huella del turismo total en el destino en 2018 de 3,12 millones de toneladas de dióxido de carbono equivalentes. Como se puede observar, la huella de carbono por mercados varía teniendo en cuenta la distancia desde el país de emisión. En la figura se han mostrado solo los principales mercados emisores a La Habana.

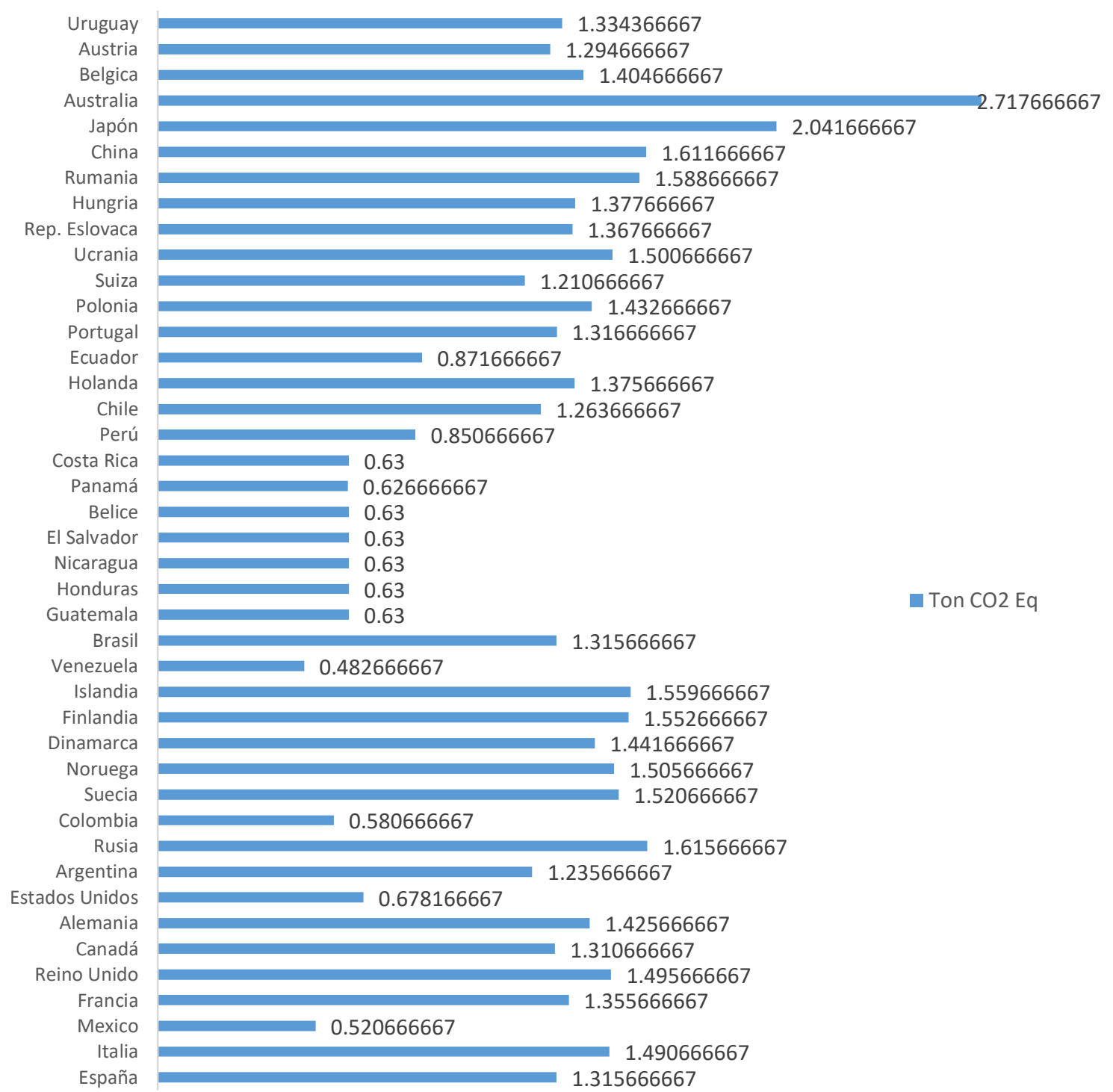

Figura 1. Huella de carbono por turista que visita La Habana de acuerdo al país de emisión. 2018

Fuente: Elaboración propia. 
Otro de los elementos fundamentales es que, los países nórdicos, aunque se encuentran en un área geográfica más cercana que España e Italia, tienen una huella de carbono superior. Esto se debe a que no existen vuelos directos desde el país de emisión y debe hacerse una conexión en un tercer país por lo que aumentan las emisiones provocadas por el viaje.

Los pronósticos de llegadas de turistas a La Habana tendrán un impacto en la huella de carbono del sector. Teniendo en cuenta que en los próximos 10 años no se produzcan cambios tecnológicos sustanciales y que el comportamiento en el destino deje una huella similar a la actual, se puede estimar que las emisiones de carbono aumentarán consistentemente con el arribo de turistas al destino como ha sucedido hasta el momento (Figura 2).

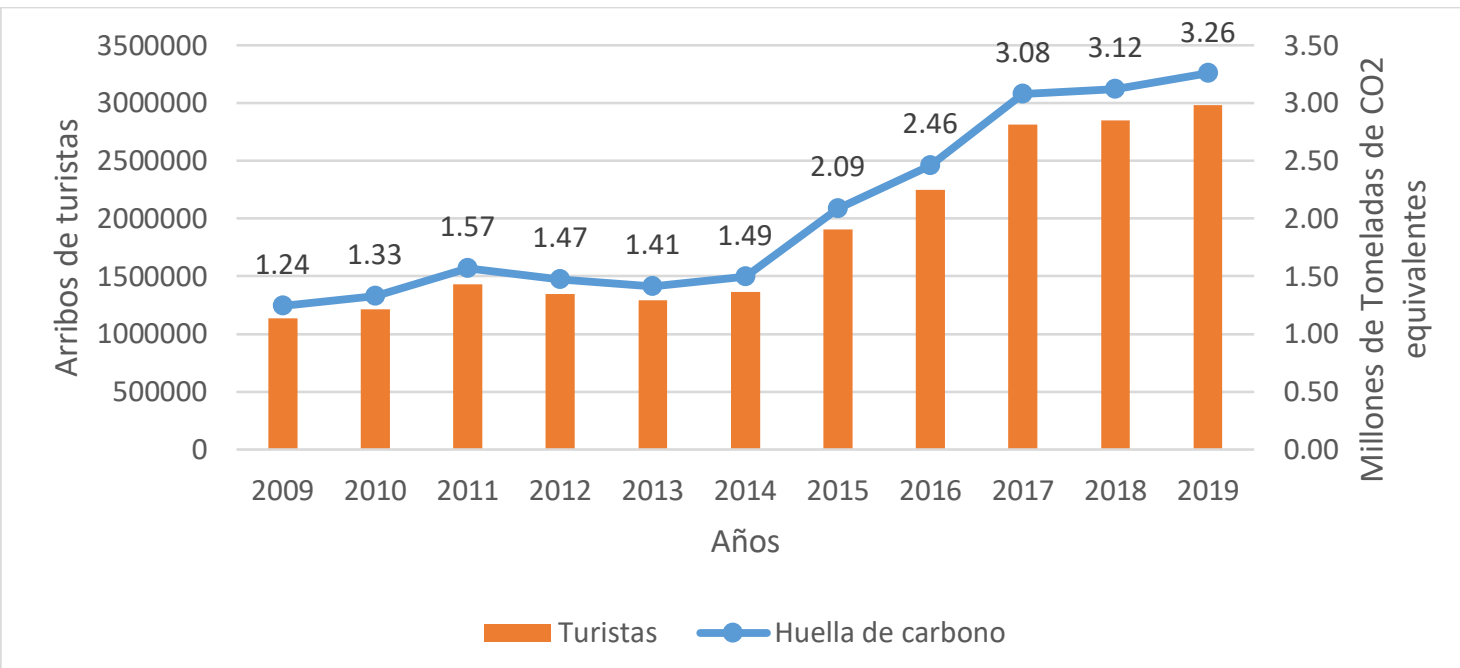

Figura 2. Comparación de los FCE entre los grupos hoteleros cubanos

Fuente: Elaboración propia.

Lo anterior estresa la necesidad de enfocar las acciones de comercialización hacia una cartera de mercados con mayor participación de países más cercanos.

\section{Factores y actores que intervienen en la disminución de la huella de carbono.}

El análisis de los factores exógenos de la actividad turística permitió conocer que con respecto a la política, Cuba en el Acuerdo de París trazó como objetivo disminuir gradualmente la huella de carbono con ayuda internacional, por lo tanto las iniciativas vinculadas con un turismo bajo en emisiones de carbono con congruentes con este compromiso, así como con los programas nacionales de enfrentamiento y mitigación al cambio climático, muchos de los que se concretan y gestionan a través de la "Tarea Vida" y las comunicaciones nacionales sobre Cambio Climático, dentro de la cual el turismo tiene un acápite. 
En cuanto a factores económicos, el país se enfrenta a un bloqueo económico, comercial y financiero que limita su actuación con respecto a la adquisición de tecnologías de elevada eficiencia energética. La infraestructura tecnológica del país por consiguiente en su mayoría obsoleta aunque se dan varios pasos de avance. Uno de los elementos más afectados y de mayor incidencia es la transportación dentro del país, con un parque de aviones de las aerolíneas nacionales ineficientes desde el punto de vista energético, autos poco modernos y en caso de los oferentes privados la situación se complejiza pues la mayoría de los autos a disposición para el turismo tienen más de 40 años en explotación. No obstante, la situación económica del país, imposibilitado de adquirir combustibles fósiles a precios competitivos, ha determinado que un desarrollo bajo en emisiones de carbono, especialmente en el turismo, sea una necesidad pues es esencial la disminución de la utilización de combustibles fósiles. En este momento el país ha realizado varios avances en los cambios de su matriz energética hacia fuentes renovables (eólica y solar fundamentalmente) las cuales poco a poco se introducen en el sector del turismo.

Un factor medular a tener en cuenta es que la posición geográfica del país y su condición de isla determina que solo se pueda acceder por avión o barco por lo cual la huella de carbono siempre será elevada.

Aunque el sector del turismo se ha insertado de a lleno en la Tarea Vida y se han tomado varias medidas con respecto a la mitigación del impacto del cambio climático, aun no hay un conocimiento profundo de los trabajadores con respecto a este tema o sobre qué es la huella de carbono y cómo disminuirla. De igual forma no existe en el país un marco regulatorio o impositivo que favorezca una reducción en las emisiones de $\mathrm{CO} 2$ en ninguno de los sectores económicos.

Desde el punto de vista endógeno, el país cuenta con una variada riqueza cultural, histórica y natural para el desarrollo de productos turísticos locales que minimicen las emisiones de carbono durante la experiencia turística. No obstante, hasta el momento no se diseñan o comercializan productos turísticos bajos en emisiones de carbono o que compensen las emisiones por desconocimiento de los actores implicados, principalmente los agentes de viaje.

Desde la demanda, se está produciendo un movimiento hacia el consumo de productos turísticos bajos en emisiones de $\mathrm{CO} 2$ lo cual es una ventaja pues existe un nicho con patrones de consumo congruentes. Otro elemento a tener en cuenta es que, como se había mencionado anteriormente, la estructura de mercados emisores a Cuba determina una mayor huella de carbono pues los turistas provienen en mayor medida de Europa, América del sur y Asia.

Con respecto a la participación de los actores, hasta el momento, no todos los actores realizan acciones en consecuencia debido al desconocimiento, principalmente de los gestores y operadores de las instalaciones y productos turísticos, mientras se puede observar un mayor conocimiento entre los gestores a nivel de país. Aunque, las acciones de mitigación a los impactos del cambio climático han sido significativos, en la generación de productos turísticos y su gestión no se han hecho avances hasta el 
momento. Las universidades, centros de investigación y otras instituciones científicas y académicas, en estos momentos comienzan a dar los primeros resultados que pueden ser aplicados como parte de la política de I+D+i en el vínculo Universidad - Empresa promovido por el Presidente del País. El resto de los actores en estos momentos (la comunidad de acogida y la prensa), ante la inexistencia de iniciativas turísticas de este tipo, no se han visto implicados aunque pueden jugar un papel fundamental en la promoción y en las acciones de Relaciones Públicas que promuevan productos turísticos bajos en emisiones de carbono. Organismos internacionales han brindado financiamiento para iniciativas que se relacionen con la mitigación del impacto del cambio climático y entre las acciones contemplen la disminución de la huella de carbono.

\section{Contextualización de las buenas prácticas internacionales a la realidad cubana}

Después de analizadas las buenas prácticas descritas en el documento de análisis sobre medidas de mitigación del cambio climático para el transporte aéreo internacional propuesto por la OMT (2009), los estudios de Can y Hongbing (2011), los estudios para un crecimiento bajo en carbono a partir de políticas transectoriales en el Estado Plurinacional de Bolivia, Colombia, Nicaragua y el Perú (Coronado et al, 2014), las oportunidades y retos de una economía turística baja en carbono para el sudeste de Inglaterra (Whittlesea, 2016), el desarrollo turístico bajo en carbono de Jiangxi (Ningrum et al, 2017), así como la evaluación de la experiencia turística baja en emisiones de carbono realizada por Jan y Lee (2019), estas se contextualizaron a la realidad cubana teniendo en cuenta el análisis realizado en la sección anterior.

En un destino turístico como Cuba al que se accede principalmente por aire y por mar, y en el que el turismo interno no se ha desarrollado de forma tan extensiva, el porciento mayor de emisiones de $\mathrm{CO} 2$ de la actividad turística proviene de la transportación aérea al destino (77,9\% de las emisiones de $\mathrm{CO} 2$ equivalentes per cápita).

Un factor a tener en cuenta es la situación económica y financiera en que se encuentra el país, por lo que desde el gobierno solo la voluntad política de negociar con las aerolíneas que viajen al destino la implementación de acciones de reducción de su huella de carbono, es la única medida válida en este sentido en el corto y mediano plazo. Estas acciones deberían estar enfocadas a la negociación de un mayor número de vuelos directos al destino desde países que ahora solo vuelan al destino a través de conexiones aéreas, la utilización de aviones con tecnología más eficiente en el consumo de combustible y de mayor porte. La aplicación de impuestos no ha sido hasta el momento en el mundo una vía efectiva para la reducción de los gases de $\mathrm{CO} 2$ de la aviación, sin embargo, de acuerdo a la OMT las políticas de incentivos para minimizar las fugas de carbono a las empresas de aviación pudiera lograr el efecto deseado.

El país tiene dos aerolíneas propias, Aerogaviota y Cubana de Aviación, las cuales tienen aviones de tecnología poco eficientes con respecto a la reducción de emisiones de gases de infecto invernadero en general. Se trabaja en este momento por obtener financiamiento 
para la modernización de la flota cubana lo cual se prevé como la única política factible de realizar en este momento.

Varios estudios internacionales han producido una serie de medidas para la reducción de la huella de carbono en destinos turísticos las cuales pueden ser aplicadas en Cuba entre las que se encuentran:

(1) Promover la utilización de medios de transporte con bajo consumo de energía o que utilicen fuentes renovables, así como la promoción del uso de medios públicos en detrimento de los privados como por ejemplo la promoción de los viajes por trenes, medios de transporte eléctricos o movidos por energía solar, las bicicletas, entre otros,

(2) Promover la realización de actividades bajas en consumo de energía, como actividades al aire libre,

(3) Establecer políticas impositivas por emisiones de carbono a los proveedores de servicios en el destino,

(4) Conformar sistemas de reducción de emisiones de carbono a partir de la implementación de tecnologías de avanzada desarrolladas por la academia,

(5) Etiquetar los productos de acuerdo a su huella de carbono,

(6) Promover el cambio en los estilos de consumo de los turistas a partir de un sistema de incentivos para el consumo de productos locales y bajos en huella de carbono.

Con respecto a las dos primeras de estas medidas, el MINTUR y los oferentes privados deben intensificar las acciones de comunicación con respecto a la promoción de un turismo bajo en carbono, lo cual es congruente con la política del Ministerio de promover un turismo más sostenible y con la Tarea Vida promovida con el Gobierno. Ya se han introducido en el transporte público de la capital algunos autobuses eléctricos, sin embargo, esto no se ha realizado en el sistema de trasporte al turismo. Priorizar la introducción de estos autobuses con esta tecnología al menos en los viajes al interior de los destinos pudiera ser una medida válida en una primera etapa. Los oferentes privados han promovido como experiencias los recorridos en bicicleta por la ciudad y hacia otros destinos, así como las caminatas y los paseos a caballo lo cual constituye una buena práctica pero que no es aún suficiente. El estímulo a los oferentes de estos productos, sean públicos o privados, facilitando los mecanismos de importación de piezas y reducción de sus obligaciones impositivas pudieran ser elementos que contribuyan al desarrollo de más productos turísticos bajos en emisiones de carbono. Se ha anunciado por parte del gobierno el desarrollo de un sistema de transporte bajo en emisiones de dióxido de carbono en la Capital el cual se encuentra en la fase aprobación.

Con respecto a las políticas impositivas, estas deben aplicarse a los mayores emisores. Sin embargo, en el caso cubano debe tenerse en cuenta que en buena medida estos emisores son los transportistas privados que cuentan con automóviles de más de 30 años en explotación, híbridos en muchos casos por no encontrar en el mercado interno las 
piezas necesarias o por no facilitarse el acceso a autos más modernos y eficientes. Estos son grandes consumidores de combustible y emisores de carbono, por lo que en el caso particular de los transportistas privados, un impuesto sobre emisiones, lejos de mejorar puede emporar la situación. Puede con ellos utilizarse una política similar a la de la aviación, emplear estímulos para la disminución en las emisiones de carbono en una primera etapa hasta que el país sea capaz de satisfacer la demanda de autos modernos a precios accesibles para los oferentes de servicios de transporte privados, momento en el que se podrían aplicar medidas impositivas.

De acuerdo con la Oficina Nacional de Estadísticas e información de Cuba (2018), el sector del turismo en 2017 solo aportó medio millón de CUC para la preservación del medio ambiente, siendo los recursos naturales el principal atractivo turístico del país. Una política de impuestos sobre la emisión de carbono sería una medida acertada en este sentido para que el sector realice una mayor contribución. Una parte específica de los ingresos derivados de los impuestos de emisión en el país en general podría destinarse a actividades de compensación de emisiones de gases de efecto invernadero relacionadas con el transporte aéreo doméstico y el turismo, y deberían existir incentivos financieros o de otra índole para la introducción a la mayor brevedad posible de biocombustibles sostenibles para la aviación y otros medios de transporte en el destino. De igual forma pudiera exigirse a los nuevos proyectos de desarrollo hotelero e inmobiliario esquemas de uso de energías renovables en su operación como el uso de paneles solares.

Una buena práctica es el etiquetado de los productos de acuerdo a su huella de carbono o generar marcas locales, promoviendo en los turistas el consumo de productos bajos en carbono. Los gobiernos locales pueden hacer una gran contribución en este sentido, etiquetando productos y generando marcas para los servicios. Otra práctica a considerar es un sistema de certificaciones ambientales que realmente estimule su obtención. Ambas acciones, el etiquetado y la certificación válida para productos de todo tipo y servicios, puede realizarse en vínculo estrecho con la academia o centros científicos para facilitar ambos procesos, especialmente la medición que puede resultar complicada para los gestores turísticos.

Otra práctica que pudiera introducirse en Cuba es la compensación, en la cual los emisores de dióxido de carbono plantan árboles o realizan actividades similares que contribuyan a compensar por las emisiones realizadas, lo cual pudiera ser una práctica positiva ya que la demanda cada vez es más consciente de los efectos ambientales de la actividad turística y prefieren tener una actitud responsable al respecto.

Una de las acciones fundamentales debe ser la capacitación de los actores que convergen en el destino turístico con respecto al turismo bajo en emisiones de carbono, su importancia, así como el rol que juega cada actor en su consecución. Debe prestarse especial atención a la capacitación con respecto al diseño y desarrollo de productos/ofertas turísticas bajas en emisiones de carbono. La prensa juega un papel fundamental en la labor de divulgación y atracción de la comunidad para que se integre 
en este desarrollo que puede beneficiarla a partir de la creación de productos locales o de $0 \mathrm{~km}$.

\section{Conclusiones}

- La presente investigación es un primer paso para lograr un desarrollo turístico bajo en emisiones de carbono en Cuba, a través de la cual se pudo estimar que la huella de carbono promedio de un turista que visita La Habana es de 1,09 tCO2eq y se pronostica la huella total de carbono del turismo se comportará en el futuro consistentemente con los arribos turísticos al país.

- Hasta el momento no existe acuerdo en la comunidad científica sobre una metodología para la medición de la huella de carbono en el turismo. No obstante, el uso de una metodología de medición en unión con el análisis de los factores que constituyen el Modelo Pentagonal del Turismo (Martín, 2010), la teoría de actores socioeconómicos convergentes para la sostenibilidad de un destino (Torres, 2017) y la identificación de buenas prácticas, permitieron proponer acciones concretas adaptadas a la realidad del destino y específicas de acuerdo a los actores implicados lo cual puede extenderse a otras investigaciones.

- Los principales elementos que influyen actualmente en la huella de carbono del turismo en Cuba son su estructura de mercados, el desconocimiento de los actores con respecto al fenómeno y el bloqueo económico, financiero y comercial de Estados Unidos. Las principales acciones que puede acometer el sector del turismo en el corto plazo es la capacitación a los diferentes actores que intervienen en el turismo, un balance entre las medidas impositivas y de estimulación para la disminución de la huella de carbono, así como la voluntad política y la cooperación internacional.

\section{Referencias bibliográficas}

Becken, S.; Patterson, M. (2006) Measuring national carbon dioxide emissions from tourism as a key step towards achieving sustainable tourism. Journal of Sustainable Tourism 14, 323-338.

Cadarso, M.Á.; Gómez, N.; López, L.A.; Tobarra, M.Á. (2016): Calculating tourism’s carbon footprint: Measuring the impact of investments. Journal of Cleaner Productions. (111), 529-537.

Cadarso, M.Á.; Gómez, N.; López, L.A.; Tobarra, M.Á.; Zafrilla, J.E. (2015) Quantifying Spanish tourism's carbon footprint: The contributions of residents and visitors: A longitudinal study. Journal of Sustainable Tourism, 23, 922-946

Can, H., \& Hongbing, D. (2011). The model of developing low-carbon tourism in the context of leisure economy. Energy Procedia, 5, 1974-1978. http://doi.org/10.1016/j.egypro.2011.03.339 
Changbo, S., y Jingjing, P. (2011). Construction of Low-carbon Tourist Attractions Based on Low-carbon Economy. Energy Procedia, 5, 759-762. http://doi.org/10.1016/j.egypro.2011.03.133

Chen, L. Q. (2010) The Trend of International Low-carbon Economy in the New Century. Journal of Zhengzhou Institute of Aeronautical Industry Management, 3, 1-12 .

Coronado, Harold, Jaime, Haider, Gamba, Paloma (2014) Crecimiento bajo en carbono: Políticas en Bolivia (Estado Plurinacional de), Colombia, Nicaragua y Perú. Estudios de Cambio Climático en América Latina. Naciones Unidas, Santiago de Chile.

Filimonau, V.; Dickinson, J.E.; Robbins, D.; Huijbregts, M.A.J. (2011): Reviewing the carbon footprint analysis of hotels: Life Cycle Energy Analysis (LCEA) as a holistic method for carbon impact appraisal of tourist accommodation. Journal of Cleaner Productions, 19, 1917-1930.

Gössling, S.; Hall, M. (2008): Swedish tourism and climate change mitigation: An emerging conflict. Scandinavian Journal of Hospitality and Tourism, 8, 141-158.

Jan, F. y Lee, T.H. (2019). The Low-Carbon Tourism Experience: The Low-Carbon Tourism Experience: A Multidimensional Scale Development. Journal of Hospitality \& Tourism Research, XX, (X), 1-29. http://doi.org/10.1177/1096348019849675

Khoo-lattimore, C y Prideaux, B., (2013). ZMET : a psychological approach to understanding unsustainable tourism mobility. Journal of Sustainable Tourism, 21(7), 1036-1048.

Kumar, S., Kusakabe, Kyoko, Shrestha, Pujan, Linh, Nguyen Khanh, y Suwanprik, Trinnawat. (2016). Sustainable urban tourism through low-carbon initiatives: Experiences from Hue and Chiang Mai. Asian Institute of Technology.

Lund-durlacher, D y Dimanche, F., (2013). Mobilities and sustainable tourism: an introduction. Journal of Sustainable Tourism, 21(4), 505-510.

Luo, J., y Zhang, M. (2011). Route Choice of low-carbon industry for global climate change: an issue of China tourism reform. Energy Procedia, 5, 2283-2288. http://doi.org/10.1016/j.egypro.2011.03.394

Martín, R. (2010): Principios, organización y práctica del turismo. Editorial Félix Varela, La Habana, Cuba.

Moscardo, G. et al., (2013). Mobilities, community well-being and sustainable tourism. Journal of Sustainable Tourism, 21(4), 532-556. 
Munday, M.; Turner, K.; Jones, C. (2013) Accounting for the carbon associated with regional tourism consumption. Tourism Management, 36, 35-44.

Ningrum, L., Hendradewi, S., y Nurbaeti, N. (2017). A study on rural low-carbon tourism development in Jiangxi. IOP Conference Series: Earth and Environmental Science. IOP Publishing.

Oficina Nacional de Estadísticas e Información (2018) Panorama ambiental Cuba 2017. La Habana, Cuba.

Organización de las Naciones Unidas (2019) "Se alcanzan niveles récord de concentración de gases de efecto invernadero en la atmósfera". Noticias ONU. $\begin{array}{llllll}\text { Accedido el } & 26 & \text { de } & \text { noviembre }\end{array}$ https://news.un.org/es/story/2019/11/146585

Organización Mundial del Turismo (2008) The United Nations Environment Programme (UNEP).

Organización Mundial del Turismo (2009) Documento de análisis sobre medidas de mitigación del cambio climático para el transporte aéreo internacional. Madrid, España.

Organización Mundial del Turismo y Organización de los Estados Americanos (2018) El turismo y los Objetivos de Desarrollo Sostenible - Buenas prácticas en las Américas. OMT, Madrid. DOI: https://doi.org/10.18111/9789284419937.

Pina, A., Ferrão, P., Fournier, J., Lacarrière, B., \& Corre, O. Le. (2017). CO2 emmission of tourist transportation in Suan Phueng Thailand. Energy Procedia, 136, 438443. http://doi.org/10.1016/j.egypro.2017.10.300

Pina, A., Ferrão, P., Fournier, J., Lacarrière, B., \& Corre, O. Le. (2018). Scenario Planning for Low Carbon Tourism City: A Case Study of Nan Assessing the feasibility of using the heat temperature function for a long-term district heat demand forecast. Energy Procedia, 152, 715-724. http://doi.org/10.1016/j.egypro.2018.09.235

Qi, Z., y Hong, L. I. U. (2011). Study on Design and Research of Tourist Souvenirs on the Background of Low-carbon Economy. Energy Procedia, 5, 2416-2420. http://doi.org/10.1016/j.egypro.2011.03.415

Rico, Anna; Martínez-Blanco, Julia; Montlleó, Marc; Rodríguez, Gustavo; Tavares, Nuno; Arias, Albert; Oliver-Solà, Jordi (2019): "Carbon footprint of tourism in Barcelona” Tourism Management, 70, 491-504 
Tang, Z., Shi, C. B., \& Liu, Z. (2011). Sustainable Development of Tourism Industry in China under the Low-carbon Economy. Energy Procedia, 5, 1303-1307. http://doi.org/10.1016/j.egypro.2011.03.226

Torres Hechavarría, Lisandra (2017) Propuesta de esquema metodológico para la evaluación de la sostenibilidad del desarrollo turístico de destinos. Caso La Habana. Editorial Universitaria. La Habana, Cuba

Tsai, K., \& Lin, T. (2018). The Carbon Impact of International Tourists to an Island Country, Sustainability, 10, 1-12. http://doi.org/10.3390/su10051386

Wang, S., Hu, Y., He, H., \& Wang, G. (2017). Progress and Prospects for Tourism Footprint Research, Sustainability, 9, 1-17. http://doi.org/10.3390/su9101847

Whittlesea, Ema Rachel (2016): “AN INVESTIGATION INTO THE OPPORTUNITIES AND CHALLENGES FOR A LOW-CARBON TOURISM ECONOMY IN THE SOUTH WEST OF ENGLAND”. PhD. Thesis. Plymouth University.

World Meteorological Organization (WMO) (2008). Climate Change and TourismResponding to Global Challenges; UNWTO: Madrid, Spain.

Yang, W. (2010). The Development of Tourism in the Low Carbon Economy, International Business Research 3(4), 212-215.

Yu, Ouyang y Tsung-Lin, Lee (2019) A study on local identity of low carbon tourism based on social exchange theory- a case study of Taiwan' s characteristic Hot Spring area., International Journal of Organizational Innovation 12(3), 355-366.

Yuan, H., Zhou, P., \& Zhou, D. (2011). What is Low-Carbon Development? A Conceptual Analysis Abstract: Energy Procedia, 5, 1706-1712. http://doi.org/10.1016/j.egypro.2011.03.290

Zhang, D., Zhen, Y., \& Zhang, E. (2016). Research on the Models of Low- Carbon Tourism based on the Sustainable Development, International Conference on Applied Social Science Research (ICASSR 2015) Proceedings, 99-102.

\section{\Ciencia}




\section{PARA CITAR EL ARTÍCULO INDEXADO.}

Torres Hechavarría, L., \& Delgado Castro, A. (2021). Claves para la disminución de la huella de carbono en el turismo: Caso Cuba. Explorador Digital, 5(1), 6-24. https://doi.org/10.33262/exploradordigital.v5i1.1487

\section{LCiencia}

El artículo que se publica es de exclusiva responsabilidad de los autores y no necesariamente reflejan el pensamiento de la Revista Explorador Digital.

El artículo queda en propiedad de la revista y, por tanto, su publicación parcial y/o total en otro medio tiene que ser autorizado por el director de la Revista Explorador Digital.
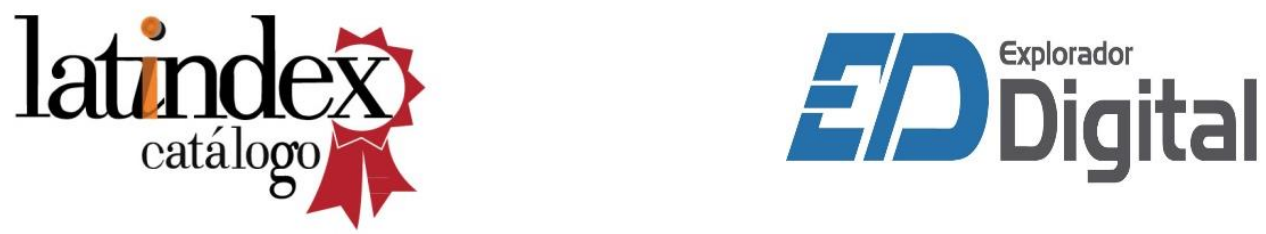